\title{
Sub-harmonic mode-locking of VCSEL with a concave external mirror
}

\author{
Tomoyuki Kato $^{\text {a) }}$, Akihiro Matsutani, Takahiro Sakaguchi, \\ and Kohroh Kobayashi \\ Precision and Intelligence Laboratory, Tokyo Institute of Technology \\ 4259-R2-21, Nagatsuta, Midori-ku, Yokohama, Kanagawa 226-8503, Japan \\ a)kato.t.ae@m.titech.ac.jp
}

\begin{abstract}
We designed a composite resonator VCSEL using a VCSEL chip and a concave external mirror for mode-locking to produce optical clock signals and demonstrated the operation experimentally. A very low threshold current was successfully realized by using a proper reflectivity of VCSEL and the external mirror and by achieving efficient feedback using a concave external mirror. Using a concave mirror with the curvature radius of $14.12 \mathrm{~mm}$, sub-harmonic active mode-locking was achieved at a repetition rate of $10.56 \mathrm{GHz}$, which corresponded to the external cavity length.
\end{abstract}

Keywords: VCSEL, mode-locked laser diode, external cavity

Classification: Photonics devices, circuits, and systems

\section{References}

[1] S. Hoogland, S. Dhanjial, A. C. Tropper, J. S. Roberts, R. Häring, R. Pashotta, F. Morier-Genoud, and U. Keller, "Passively mode-locked diode-pumped surface-emitting semiconductor laser," IEEE Photon. Technol. Lett., vol. 12, no. 9, pp. 1135-1137, Sept. 2000.

[2] U. Fliedler and K. J. Ebeling, "Design of VCSEL's for feedback insensitive data transmission and external cavity active mode-locking," IEEE J. Sel. Topics Quantum Electron., vol. 1, no. 2, pp. 442-450, June 1995.

[3] K. Jasim, Q. Zhang, A. V. Nurmikko, E. Ippen, A. Mooradian, G. Carey, and W. Ha, "Picosecond pulse generation from passively modelocked vertical cavity diode laser at up to $15 \mathrm{GHz}$ pulse repetition rate," Electron. Lett., vol. 40, no. 1, pp. 34-36, Jan. 2004.

\section{Introduction}

Signal processing speed of LSIs is predicted to exceed $10 \mathrm{GHz}$ within a few years and such a large increase in speed will further continue. This progress will induce difficulty in transmitting signals among LSIs on a circuit board. Optical interconnection is expected to realize high speed and large capacity signal transmission. Besides the signal transmission, clock distribution is also an important issue for the inter-chip optical interconnection. Vertical 
cavity surface emitting lasers (VCSELs) are considered to be a promising light source for the signal as well as clock generation because of the low power consumption. As far as the optical clock pulse generation, mode locking scheme seems to be a powerful tool for generating high repetition rate optical pulses, because the repetition frequency is, in principle, free from the direct modulation bandwidth of the VCSEL. Among mode-locked VCSELs with an external mirror investigated so far are a VCSEL with a concave mirror [1] and one with a single mode fiber [2]. The former device was optically pumped which resulted in the large cavity size and a low repetition rate of $4.4 \mathrm{GHz}$. The latter used a single mode fiber for the external cavity and pumped by injection current. The repetition rate is still low at less than $1 \mathrm{GHz} .15 \mathrm{GHz}$ repetition rate has been achieved in a VCSEL with a separate DBR mirror [3]. The threshold current, however, was quite high, i.e., $400 \mathrm{~mA}$.

The reflectivity of VCSEL DBR mirrors and the external concave mirror were designed for a low threshold current and a large number of axial mode oscillation. We present low threshold current mode-locked VCSEL with a concave external mirror for the first time. Threshold current of as low as $0.5 \mathrm{~mA}$ was obtained. Light output at the repetition rate of $10 \mathrm{GHz}$ was observed by sub-harmonic active current modulation.

\section{Design consideration}

Figure 1 shows the fundamental configuration of our device which consists of a VCSEL chip and a concave external mirror. The repetition rate of the mode-locked pulse is determined by the external cavity length. It is close to the curvature radius of the concave mirror, because we used the spherical mirror and the launching point and the focal point are the center of the sphere. A reflectivity of the top DBR mirror of the VCSEL, which couples

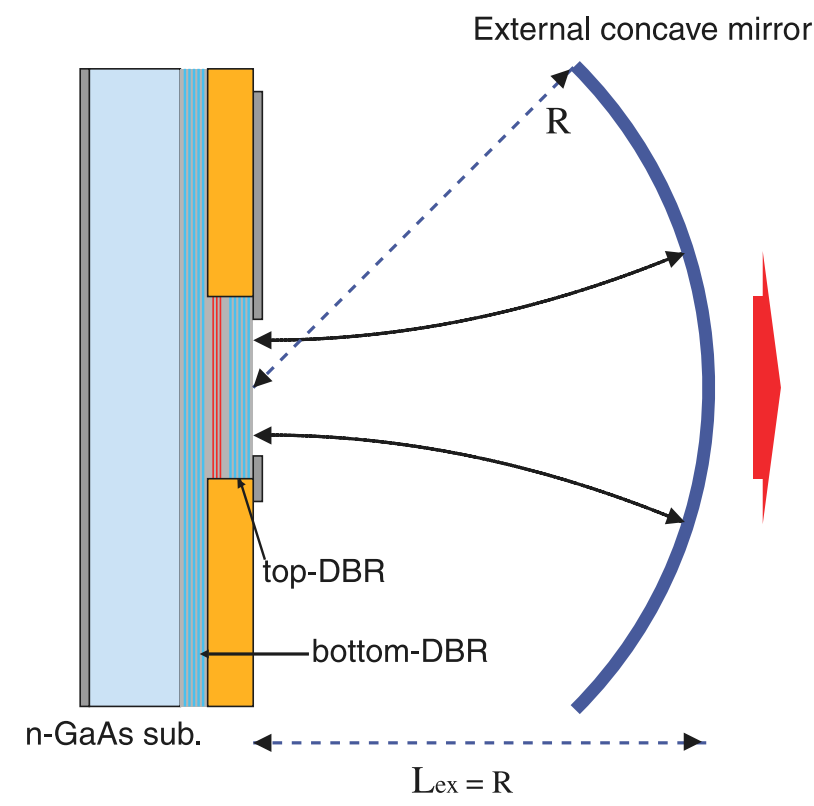

Fig. 1. Schematic of the composite resonator VCSEL using an external concave mirror. 
the VCSEL cavity and the external cavity, is a critical design issue, because the lower reflectivity will lead to a shorter optical pulse through a larger number of the external cavity axial mode to be mode-locked. From a view point of a low threshold current, however, the top DBR mirror reflectivity should be as high as possible.

In this design, we preferred a low threshold current rather than a short optical pulse width. The top DBR mirror reflectivity was designed to be $96 \%$, by which the total reflectivity of the composite mirror is assumed to be higher than $99 \%$. With the high top DBR mirror reflectivity, the number of the external cavity mode to be mode-locked is estimated to be fairly small, two to four.

\section{Fabrication}

We fabricated $980 \mathrm{~nm}$ top-emitting VCSEL chip. The active layer was InGaAs/GaAs triple QW. The top DBR mirror had 16 pairs of $\mathrm{Al}_{0.90} \mathrm{Ga}_{0.10} \mathrm{As} /$ GaAs layers. It was not enough to observe the lasing oscillation without the external mirror for the injection current up to about $10 \mathrm{~mA}$. The $30 \mu \mathrm{m}$ square mesa was formed by photolithography and ICP-etching. The carrier confinement was done by oxidation of an AlAs thin layer. The oxidation aperture was about $5 \mu \mathrm{m}$. The polyimide was spun on and p- and n-electrode were evaporated on the mesa and wafer backside, respectively.

A concave mirror of $14.12 \mathrm{~mm}$ curvature radius was made by evaporating the 6 pair $\mathrm{SiO}_{2} / \mathrm{Ta}_{2} \mathrm{O}_{5}$ DBR layers on a commercially available concave lens. The reflectivity was set to be about $97 \%$.

\section{Static characteristics}

By adjusting the mutual position of the VCSEL and the concave mirror, the lasing oscillation was achieved with a very low threshold current of $0.5 \mathrm{~mA}$ as shown in Fig. 2. It is almost the same as that of the normal VCSEL. The low threshold current indicates that the effective reflectivity of the top-DBR and the external mirror combination is sufficiently high. This also suggests that the efficient feedback is obtained.

From the spectrum response, the single transverse mode was confirmed, although the multi-transverse mode was observed in case of the misaligned feedback. The axial displacement tolerance was about $\pm 40 \mu \mathrm{m}$, which corresponded to the repetition rate change of $\pm 30 \mathrm{MHz}$. The coupling efficiency of the reflected light to the VCSEL is approximately expressed by

$$
\eta=\frac{1}{\sqrt{1+\left(\frac{\lambda \delta L}{\pi w_{0}^{2}}\right)^{2}}},
$$

where $w_{0}$ is the spot size of the VCSEL and $\delta L$ is the axial displacement from the optimum position. From the axial tolerance of $\pm 40 \mu \mathrm{m}$, the minimum coupling efficiency of $50 \%$ was estimated by using the spot size value of $\sim 3 \mu \mathrm{m}$ in Eq. (1). From the RF spectrum response of DC operated com- 


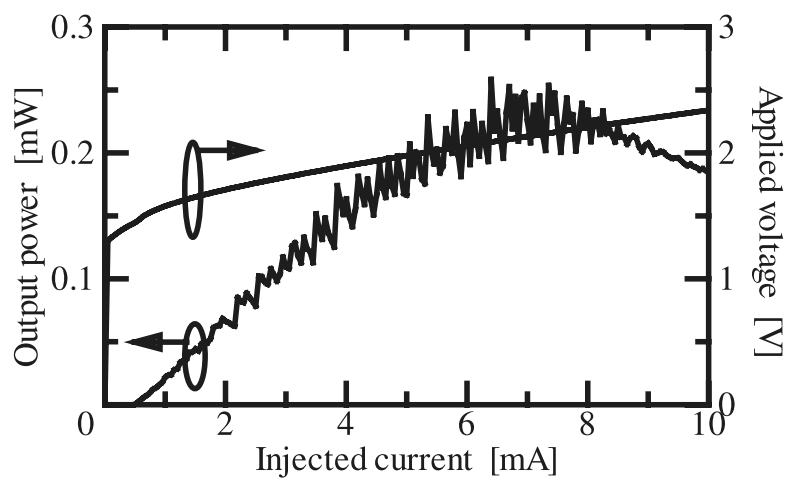

Fig. 2. I-L and I-V characteristics of the composite resonator VCSEL.

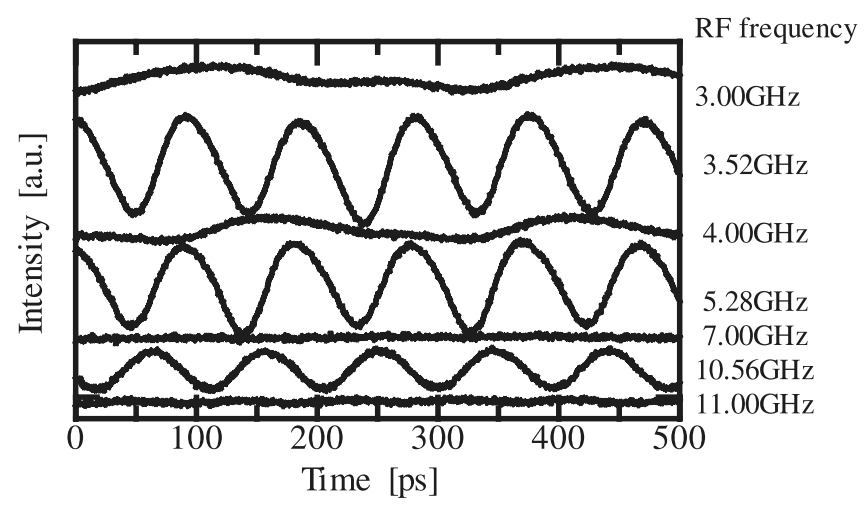

Fig. 3. Waveform of $10.56 \mathrm{GHz}$ mode-locked pulse with sub-harmonic synchronization.

posite resonator VCSEL, it was confirmed that the external cavity length corresponds to $10.56 \mathrm{GHz}$.

\section{Sub-harmonic active mode-locking}

In addition to the DC bias current, we applied RF current to the VCSEL to investigate the active mode-locking of this composite resonator VCSEL. The time responses are shown in Fig. 3 for various RF frequencies. They were observed using a photodiode with a $16 \mathrm{GHz}$ bandwidth. The DC bias current was $6 \mathrm{~mA}$. For the RF frequencies of $3.52,5.28$ and $10.56 \mathrm{GHz}$, modulated light output waveforms of sinusoidal wave-like were observed, while no modulation was observed for the other RF frequencies. The frequencies of 3.52 and $5.28 \mathrm{GHz}$ corresponded exactly to a third and a half of $10.56 \mathrm{GHz}$, i.e. the sub-harmonic frequencies of $10.56 \mathrm{GHz}$. These results seem to suggest that the observed modulated light output is caused by the active sub-harmonic mode-locking of the external cavity VCSEL.

\section{Conclusion}

We have demonstrated a low threshold current lasing operation of a composite resonator VCSEL by combining a properly designed VCSEL chip and a concave mirror. A sub-harmonic active mode-locking was suggested to be 
occurred by the light output waveform observation under RF injection with a sub-harmonic frequency of the external cavity, i.e. $10.56 \mathrm{GHz}$. This scheme appears to be promising for the high-speed optical clock pulse generation.

\section{Acknowledgments}

This work was supported in part by the JST Research "Development of Ultra-High-Frequency Clock Element Using Nano Machining Technology" promoted by JST Innovation Satellite Niigata. 